\title{
Sudden cardiac death as a naturally-occurring ventricular hypertrophy in Macaca fascicularis
}

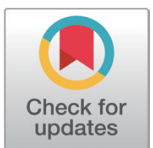

Received: Aug 30, 2021

Revised: Sep 10, 2021

Accepted: Sep 13, 2021

${ }^{\dagger}$ These authors equally contributed to this work.

${ }^{*}$ Corresponding author

Sung Soo Kim

Department of Internal Medicine

and Cardiovascular Center, Chosun

University Hospital, University of

Chosun College of Medicine, Gwangju

61453 , Korea

Tel: 82-62-220-3240

E-mail: kholywater@naver.com

Youngjeon Lee

National Primate Research Center,

Korea Research Institute of Bioscience

and Biotechnology, Ochang 28116,

Korea

Tel: 82-43-240-6316

E-mail: neurosci@kribb.re.kr

Kyung Seob Lim

Futuristic Animal Resource \& Research Center, Korea Research Institute of

Bioscience and Biotechnology, Ochang 28116, Korea
Hyeon-Gu Yeo ${ }^{1,4, \dagger}$, Junghyung Park ${ }^{1, \dagger}$, Hyun Kuk Kim ${ }^{3, \dagger}$, Joo Myung Lee ${ }^{5}$, Bon-Sang Koo ${ }^{1}$, Jeong-Hee $\mathrm{An}^{1}$, Chang-Yeop Jeon ${ }^{1}$, You Jung $\mathrm{An}^{1}$, Won Seok Choi ${ }^{1}$, Sung-Hyun Park ${ }^{1}$, Jincheol Seo ${ }^{1}$, Jinyoung Won ${ }^{1}$, Keonwoo Kim ${ }^{1,4}$, Jiyeon $\mathrm{Cho}^{1}$, Yu Gyeong Kim${ }^{1}$, Minji Kim", Jung Bae Seong ${ }^{1}$, Jae-Won Huh ${ }^{1}$, Sung Soo Kim ${ }^{3, *}$, Youngjeon Lee ${ }^{1, *}$, Kyung Seob Lim ${ }^{2, *}$

${ }^{1}$ National Primate Research Center, Korea Research Institute of Bioscience and Biotechnology, Ochang 28116, Korea

${ }^{2}$ Futuristic Animal Resource \& Research Center, Korea Research Institute of Bioscience and Biotechnology, Ochang 28116, Korea

${ }^{3}$ Department of Internal Medicine and Cardiovascular Center, Chosun University Hospital, University of Chosun College of Medicine, Gwangju 61453, Korea

${ }^{4}$ Department of Functional Genomics, KRIBB School of Bioscience, Korea University of Science and Technology, Daejeon 34141, Korea

${ }^{5}$ Division of Cardiology, Department of Internal Medicine, Heart Vascular Stroke Institute, Samsung Medical Center, Sungkyunkwan University School of Medicine, Seoul 06351, Korea

\section{Abstract}

Naturally occurring left ventricular hyperplasia is a rare but lethal disease. There are very few reports of this cardiac disease in captive nonhuman primates. In a colony of Macaca mulatta (Rhesus monkey) at California National Primate Research Center, a large number of rhesus macaques were diagnosed by autopsy with naturally occurring left ventricular hypertrophy without obvious underlying diseases over a 22-year period. The confirmatory diagnosis of ventricular hypertrophy was based on findings of notable left ventricular concentric hypertrophy with reduced left ventricular lumen, which is very similar to human ventricular hypertrophy cases. This report discusses an 11-year-old Macaca fascicularis monkey (Cynomolgus monkey, crab-eating macaque), weighing $2.95 \mathrm{~kg}$, that was presented for enrollment in a pharmacokinetic (PK) study. During the PK experiment, the monkey died following a sudden decrease in percutaneous oxygen saturation and heart rate. Gross and histological examinations of the heart were performed. On gross pathology, the left ventricular wall was thickened, and the chamber lumen was reduced. In histopathological examination using hematoxylin-eosin and Masson-trichrome stains, fibrosis and myocyte disarray were not observed, but an increased cell density, compared to the normal heart, was confirmed. The autopsy results confirmed left ventricular hyperplasia as the major cause of death.

Keywords: non-human primate; autopsy; hypertrophy, left ventricular; sudden cardiac death; pathology 
Tel: +82-43-240-6308

E-mail: dvmlim96@kribb.re.kr

Copyright @ 2021 Research Institute of Veterinary Medicine, Chungbuk National University. This is an Open Access article distributed under the terms of the Creative Commons Attribution Non-Commercial License (http://creativecommons.org/ licenses/by-nc/4.0/) which permits unrestricted non-commercial use, distribution, and reproduction in any medium, provided the original work is properly cited.

ORCID

Hyeon-Gu Yeo

https://orcid.org/0000-0002-6600-6291

Junghyung Park

https://orcid.org/0000-0002-4967-8512

Hyun Kuk Kim

https://orcid.org/0000-0002-4554-041X

Joo Myung Lee

https://orcid.org/0000-0002-2178-4014

Bon-Sang Koo

https://orcid.org/0000-0003-0799-9497

Jeong-Hee An

https://orcid.org/0000-0002-7663-550X

Chang-Yeop Jeon

https://orcid.org/0000-0002-8229-7068

You Jung An

https://orcid.org/0000-0003-1528-8054

Won Seok Choi

https://orcid.org/0000-0002-4123-9443

Sung-Hyun Park

https://orcid.org/0000-0001-5477-2901

Jincheol Seo

https://orcid.org/0000-0001-8040-1944

Jinyoung Won

https://orcid.org/0000-0001-5112-6736

Keonwoo Kim

https://orcid.org/0000-0002-5717-8497

Jiyeon Cho

https://orcid.org/0000-0001-5886-7748

Yu Gyeong Kim

https://orcid.org/0000-0003-4907-3756

Minji Kim

https://orcid.org/0000-0001-6660-0197

Jung Bae Seong

https://orcid.org/0000-0001-7423-0165

Jae-Won Huh

https://orcid.org/0000-0001-5845-939X

Sung Soo Kim

https://orcid.org/0000-0002-5190-227X

Youngjeon Lee

https://orcid.org/0000-0002-5387-3441

Kyung Seob Lim

https://orcid.org/0000-0002-9117-887X

Conflict of Interest

The authors declare no potential conflict of interest.

\section{Acknowledgements}

This study was supported by grants from the Basic Science Research Program

through the National Research Foundation of Korea (NRF) funded by the Ministry of

Education, Science and Technology (NRF2020R1C1C1003990) and the KRIBB

(Korea Research Institute of Bioscience and Biotechnology) Research Initiative Program (KGM4562121), Korea.

\section{INTRODUCTION}

Naturally occurring hypertrophic cardiomyopathy can occur in many other species besides humans. In humans, about $50 \%$ of patients with hypertrophic cardiomyopathy have a predisposing genetic disorder, characterized by left ventricular hypertrophy unexplained by secondary causes and a nondilated left ventricle with preserved or increased ejection fraction [1]. Although rare, spontaneous occurrence of left ventricular hypertrophy has been reported in laboratory rhesus macaques (Macaca mulatta) [2].

This case is the first case report in Korea of an experimental nonhuman primate that died due to left ventricular hyperplasia.

\section{CASE REPORT}

In this Pharmacokinetic (PK) study, adult female (11-year-old) Cynomolgus monkeys ( $\mathrm{Ma}$ caca fascicularis) were used as the research object. This PK experiment using non-human primates was approved by the Korea Research Institute of Bioscience and Biotechnology Institutional Animal Care and Use Committee (Approval No. KRIBB-AEC-21105), and was reported in compliance with the The ARRIVE guidelines (Animal Research: Reporting of In Vivo Experiments). The monkeys were maintained in individual indoor cages at the National Primate Research Center at the Korea Research Institute of Bioscience and Biotechnology (KRIBB) The monkeys were fed commercial monkey chow (Teklad 2050 TM, Envigo, Indianapolis, IN, USA) supplemented with various fruits, and were provided water ad libitum. Animal health monitoring comprised microbiological tests for B virus, simian retrovirus (SRV), simian immunodeficiency virus (SIV), simian virus 40 (SV40), and simian T-cell lymphotropic virus (STLV) once a year. The results of these health monitoring tests were all negative.

The monkey in question was enrolled in a safety evaluation trial of a new drug developed for the treatment of Parkinson's disease. On the procedure day, the animals were anesthetized with ketamine (5 mg/kg, Yuhan Pharmacy, Seoul, Korea) and atropine $(0.04 \mathrm{mg} / \mathrm{kg}$, Jeil Pharmacy, Daegu, Korea), and anesthesia was maintained with 1\%-2\% isoflurane (Hana Pharmacy, Hwaseong, Korea) in $\mathrm{O}_{2}$ at a flow rate of $2 \mathrm{~L} /$ minute using an anesthesia machine (Royal Medical, Seoul, Korea). Heart rate, respiration rate, saturation of percutaneous oxygen monitoring and body temperature were continuously monitored throughout the study.

The monkey was fixed after general anesthesia and the drug was administrated to the cisterna magna using an automatic injector. After 1 hour of drug injection, a sudden decrease in heart rate and saturation of percutaneous oxygen was observed on the patient monitor, and epinephrine $(0.5 \mathrm{mg} / \mathrm{kg}$, intravenous injeciton) and chest compression were performed immediately; however, the monkey did not recover and died.

After death, an autopsy was performed, and abnormal findings were observed in the heart. In gross lesions, the ventricular wall was thickened and the lumen was reduced (Fig. 1). The extracted heart tissue was stained with hematoxylin-eosin and masson-trichrome stain. Fibrosis and myocyte disarray were not observed under histopathological examination, but it was 


\section{Ethics Approval}

Animal experiment was approved by the Korea Research Institute of Bioscience and Biotechnology Institutional Animal Care and Use Committee (Approval Number: KRIBBAEC-21105).

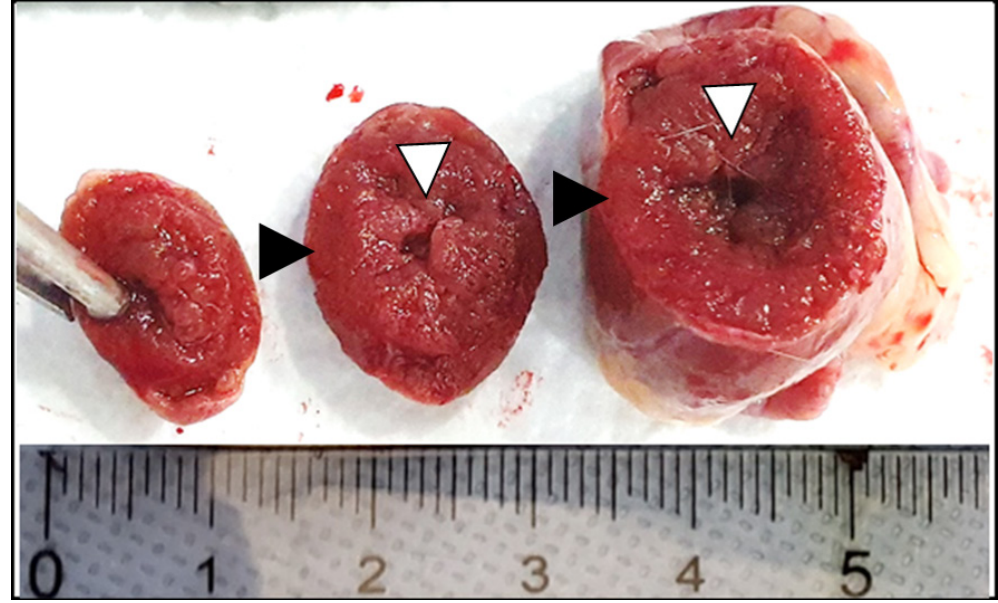

Fig. 1. Cross sectional gross findings. A markedly increased ventricular wall thickness (black arrow head) with consequent narrowing of ventricular lumen (white arrow head) was observed.

confirmed that cell density increased compared to the normal heart (Fig. 2).

\section{DISCUSSION}

Macaca fascicularis (Cynomolgus monkeys, an Old World Monkey) have been widely used as the best standard animal model in preclinical studies for new drug development because of their physiological and genetic similarity to humans $[3,4]$. In Korea, Cynomolgus monkeys are commonly used as a very important and useful experimental animal for drug development. Currently, this monkey is widely used in the research of infectious diseases such as pandemic SARS-CoV-2.

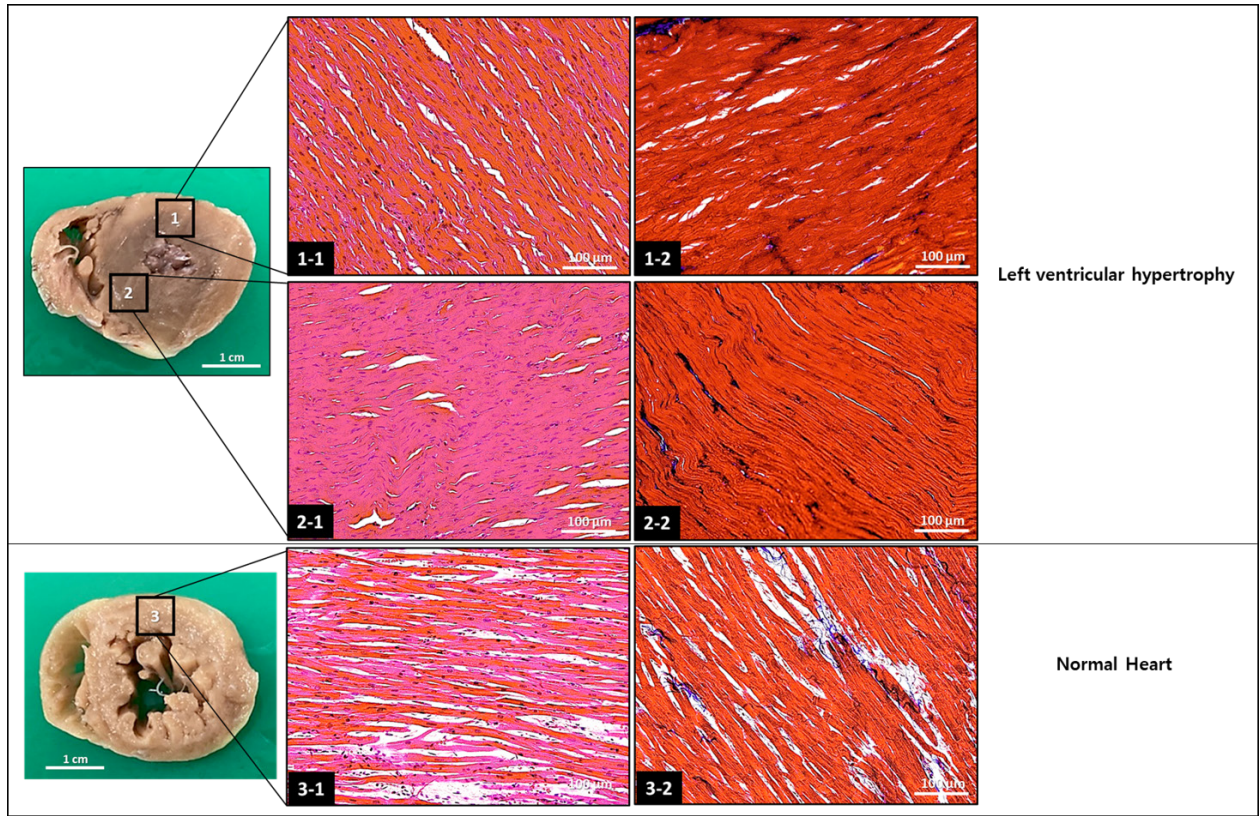

Fig. 2. Histopathological appearance of the formalin-fixed hypertrophic heart. The tissue sections were stained with hematoxylin-eosin (1-1, 2-1, and 3-1) and Mason-trichrome (1-2, 2-2, and 3-2). The hypertrophic heart show hypercellularity when compared with the normal heart. 
In humans, heart failure is a leading cause of death worldwide [5-8]. Left ventricular hypertrophy is defined as an increase in the mass and wall thickness of the left ventricle. Left ventricular hypertrophy is the most potent reason for adverse cardiac events, and is an important risk factor for sudden death, heart failure, stroke and arrhythmia [9-11]. Therefore, left ventricular hypertrophy is commonly associated with sudden cardiac death. In studies on human patients, left ventricular hypertrophy has been found to be highly related to heredity [1, 12-15].

Except for special experimental purposes such as congenital diseases experiments, the use of healthy animals without congenital diseases is an important factor in experiments using laboratory animals. Because the monkey's cardiac anatomy is similar to that in humans, non-human primates are a pivotal laboratory animal model in cardiovascular research, and thus, the selection of nonhuman primates with normal heart conditions is very important.

In this case, an autopsy was conducted due to the occurrence of sudden death during the pharmacokinetic experiment. This fatal disease was confirmed by autopsy in our experiment.

In cases of this condition, myocyte disarray and hypertrophy, fibrotic tissue deposition, nuclear atypia may be observed, but not necessarily $[14,16,17]$. In this case, only an increase in cell density was observed. However, left ventricular hypertrophy was confirmed by ventricular hypertrophy and reduced ventricular lumen in the heart.

Non-induced (Naturally-occuring) hypertrophic cardiomyopathy has previously been reported in a variety of species of feline, swine, and other monkey species [2, 18-20]. In humans, specific diagnostic criteria have been established, but there are no exact diagnostic criteria for Cynomolgus monkeys. We believe that it would be important to create a standard by accumulating necropsy data from cases of this disease observed monkeys.

In conclusion, echocardiography, cardiac computed tomography and/or cardiovascular magnetic resonance imaging are needed for the selection of healthy experimental non-human primates. Since hypertrophic cardiomyopathy can be inherited, particular caution is required in experimental monkeys used for breeding.

\section{REFERENCES}

1. Olivotto I, Girolami F, Ackerman MJ, Nistri S, Bos JM, Zachara E, Ommen SR, Theis JL, Vaubel RA, Re F, Armentano C, Poggesi C, Torricelli F, Cecchi F. Myofilament protein gene mutation screening and outcome of patients with hypertrophic cardiomyopathy. Mayo Clin Proc 2008;83:630-638.

2. Reader JR, Canfield DR, Lane JF, Kanthaswamy S, Ardeshir A, Allen AM, Tarara RP. Left ventricular hypertrophy in rhesus macaques (Macaca mulatta) at the California National Primate Research Center (1992-2014). Comp Med 2016;66:162-169.

3. Uno Y, Uehara S, Yamazaki H. Utility of non-human primates in drug development: comparison of non-human primate and human drug-metabolizing cytochrome P450 enzymes. Biochem Pharmacol 2016;121:1-7.

4. Phadke RS, Vaideeswar P, Mittal B, Deshpande J. Hypertrophic cardiomyopathy: an autopsy 
analysis of 14 cases. J Postgrad Med 2001;47:165-170.

5. Roger VL. Heart failure epidemic: it's complicated. Circulation 2018;138:25-28.

6. Yamanaka S, Sakata Y, Nochioka K, Miura M, Kasahara S, Sato M, Aoyanagi H, Fujihashi T, Hayashi H, Shiroto T, Sugimura K, Takahashi J, Miyata S, Shimokawa H, Investigators C. Prognostic impacts of dynamic cardiac structural changes in heart failure patients with preserved left ventricular ejection fraction. Eur J Heart Fail 2020;22:2258-2268.

7. Mortazavi M. Sudden cardiac death in young athletes. Adv Pediatr 2013;60:201-215.

8. Calore C, De Bortoli M, Romualdi C, Lorenzon A, Angelini A, Basso C, Thiene G, Iliceto S, Rampazzo A, Melacini P. A founder MYBPC3 mutation results in HCM with a high risk of sudden death after the fourth decade of life. J Med Genet 2015;52:338-347.

9. Gradman AH, Alfayoumi F. From left ventricular hypertrophy to congestive heart failure: management of hypertensive heart disease. Prog Cardiovasc Dis 2006;48:326-341.

10. Lip GYH, Coca A, Kahan T, Boriani G, Manolis AS, Olsen MH, Oto A, Potpara TS, Steffel J, Marín F, de Oliveira Figueiredo MJ, de Simone G, Tzou WS, En Chiang C, Williams B. Hypertension and cardiac arrhythmias: executive summary of a consensus document from the European Heart Rhythm Association (EHRA) and ESC Council on Hypertension, endorsed by the Heart Rhythm Society (HRS), Asia-Pacific Heart Rhythm Society (APHRS), and Sociedad Latinoamericana de Estimulacion Cardiaca y Electrofisiologia (SOLEACE). Eur Heart J Cardiovasc Pharmacother 2017;3:235-250.

11. Camici PG, Tschöpe C, Di Carli MF, Rimoldi O, Van Linthout S. Coronary microvascular dysfunction in hypertrophy and heart failure. Cardiovasc Res 2020;116:806-816.

12. Maron BJ, Maron MS, Semsarian C. Genetics of hypertrophic cardiomyopathy after 20 years: clinical perspectives. J Am Coll Cardiol 2012;60:705-715.

13. Seidman CE, Seidman JG. Identifying sarcomere gene mutations in hypertrophic cardiomyopathy: a personal history. Circ Res 2011;108:743-750.

14. Maron BJ. Hypertrophic cardiomyopathy: a systematic review. JAMA 2002;287:1308-1320.

15. Ingles J, Burns C, Barratt A, Semsarian C. Application of genetic testing in hypertrophic cardiomyopathy for preclinical disease detection. Circ Cardiovasc Genet 2015;8:852-859.

16. Varma PK, Neema PK. Hypertrophic cardiomyopathy: part 1 - introduction, pathology and pathophysiology. Ann Card Anaesth 2014;17:118-124.

17. Borer JS. Left ventricular hypertrophy in hypertrophic cardiomyopathy: what's in a phenotype? J Am Coll Cardiol 2004;44:406-408.

18. Huang SY, Tsou HL, Chiu YT, Shyu JJ, Wu JJ, Lin JH, Liu SK. Heritability estimate of hypertrophic cardiomyopathy in pigs (Sus scrofa domestica). Lab Anim Sci 1996;46:310-314.

19. Kittleson MD, Meurs KM, Munro MJ, Kittleson JA, Liu SK, Pion PD, Towbin JA. Familial hypertrophic cardiomyopathy in maine coon cats: an animal model of human disease. Circulation 1999;99:3172-3180.

20. Ueda Y, Kovacs S, Reader R, Roberts JA, Stern JA. Heritability and pedigree analyses of hypertrophic cardiomyopathy in rhesus macaques (Macaca mulatta). Front Vet Sci 2021;8:540493. 